\title{
IMPORTANCE OF COENZYME Q10 IN CARDIOVASCULAR DISEASE
}

\author{
K. Vijay Kumar
}

Coenzyme Q10 (CoQ10), or ubiquinone, is an electron carrier in mitochondria and plays a key role in ATP synthesis. It is also thought to have antioxidant effects and may stabilize LDL molecules. Low levels of CoQ1O have been observed in both serum and myocardial tissue of patients with various types of cardiovascular disease.CoQ10 has shown positive effects in coronary artery disease [1,2], heart failure [3,7], hypertension $[8,11]$ and even in cardiotoxicity induced by anti-cancer drugs [12]. Are the doctors who are prescribing CoQ10 wasting their patients' money, or are the conventional doctors overlooking an effective treatment?

Supplementation with CoQ10 has been clinically tested in various disease states, more so in cardiovascular diseases in more than 200 randomized controlled trials (RCT) as listed on Medline. Although results have been mixed, none of these trials have reported serious adverse effects $^{1-11}$. A few incidences of mild gastrointestinal discomfort have been registered, most probably due to the vegetable solvent in the capsule and not by CoQ10 per se. Most of the trials studied favorable effects on surrogate endpoints, the impact of CoQ10 on hard endpoints, such as mortality, has long remained unclear. Fortunately, more clarity in this respect was provided by Q-SYMBIO trial ${ }^{13}$.The Q-SYMBIO trial has shown that there was a significant improvement in NYHA functional class by at least one grade in $58 \%$ of participants, and $43 \%$ relative reduction in the primary outcome (HR 0.5; 95\% CI 0.32-0.80, $\mathrm{P}=0.003$ ) in CoQ10 group. Although the majority of studies of CoQ10 have shown beneficial effects, few trials reported negative results ${ }^{6,7}$.One possible explanation for the conflicting results is that there were differences in nutritional status among the different study populations.CoQ10 has been

Article received on 01 JAN 2017, published on 31JAN 2017

K. Vijay Kumar ${ }^{1}$

${ }^{1}$ AdditionalProfessor,DepartmentofClinicalPharmacology

\&Therapeutics, NIMS ,India

Corresponding Author: K. Vijay Kumar

Email: vijaykultala@gmail.com acknowledged in a previous American College of Cardiology/American Heart Association HF guidelines from 2005 to have a possible positive effect in some studies, but supplementation was not recommended until more robust data was available [14].

Present study has demonstrated that addition of Coenzyme Q10 at $100 \mathrm{mg} / \mathrm{d}$ has an additive effect with high dose statins in decreasing oxidative stress. Relatively small population size in the majority of the trials including the present trial raises concern about reproducibility and generalizability. While the author claims that CoQ10 is "safe and well-tolerated," the small study population makes elucidation of safety and the potential for infrequent but serious adverse effects with more widespread use difficult to discern. Hence, it may be reasonable to include CoQ10 as part of a treatment program for cardiovascular diseases especially in heart failure, because it is safe and, does not appear to interact with cardiac medications.

\section{REFERENCES:}

1. Singh RB, Neki NS, Kartikey K, Pella D, Kumar A, et al. (2003) Effect of coenzyme Q10 on risk of atherosclerosis in patients with recent myocardial infarction. Mol Cell Biochem 246: 75-82.

2. Kamikawa T, Kobayashi A, Yamashita T, Hayashi H, Yamazaki N. Effects of coenzyme Q10 on exercise tolerance in chronic stable angina pectoris. Am J Cariol 1985;56:247-51.

3. Baggio E, et al. Italian multicenter study on the safety and efficacy of coenzyme QIO as adjunctive therapy in heart failure (interim analysis). ClinInvestig. 1993;71:S145-S149.

4. Morisco---- C, et al. Effect of coenzyme QIO in patients with congestive heart failure: a long-term multicenter randomized study. ClinInvestig. 1993;71:S134-S136. 
5. Berman M, et al. Coenzyme QIO in patients with endstage heart failure awaiting cardiac transplantation: a randomized, placebo-controlled study. ClinCardiol. 2004;27:295-299.

6. Khatta M, et al. The effect of coenzyme QIO in patients with congestive heart failure. Ann Intern Med. 2000;132:636- 640.

7. Watson PS, et al. Lack of effect of coenzyme $Q$ on left ventricular function in patients with congestive heart failure. JAmCollCardiol. 1999;33:1549-155.

8. Rosen feldt FL, Haas SJ, Krum H, Hadj A, Ng K, et al. (2007) Coenzyme Q10 in the treatment of hypertension: a meta-analysis of the clinical trials. J Hum Hypertens 21: 297-306.

9. Digiesi V, Cantini F, Brodbeck B. Coenzyme Q10 in essential hypertension. Mol Aspects Med 1994;15(Suppl):257S-63S.

10. Langsjoen $P$, et al. Treatment of essential hypertension with coenzyme Q10. Mol Aspects Med 1994;15:265-72.
11. Digiesi V, Cantini F, Bisi G, et al. Mechanism of action of coenzyme Q10 in essential hypertension. CurrThes Res 1992;51:668-72.

12. Greenlee H, Shaw J, Lau YK, Naini A, Maurer M (2012) Lack of effect of coenzyme q10 on doxorubicin cytotoxicity in breast cancer cell cultures. Integr Cancer Ther 11: 243-250.

13. Mortensen, S.A., et al., The effect of coenzyme Q10 on morbidity and mortality in chronic heart failure: results from Q-SYMBIO: a randomized double-blind trial. JACC Heart Fail, 2014. 2(6): p. 641-9.

14. Hunt S.A., Abraham W.T., Chin M.H., et al. (2005) ACC/AHA 2005 guideline update for the diagnosis and management of chronic heart failure in the adult. A Report of the American College of Cardiology/American Heart Association Task Force on Practice Guidelines. J Am CollCardiol 46:e1-e82. 